\title{
Extremal Betti Numbers of Vietoris-Rips Complexes
}

\author{
Michael Goff
}

Received: 17 October 2009 / Revised: 10 July 2010 / Accepted: 10 July 2010 /

Published online: 4 August 2010

(C) Springer Science+Business Media, LLC 2010

\begin{abstract}
Upper bounds on the Betti numbers over an arbitrary field of Vietoris-Rips complexes are established, and examples of such complexes with large Betti numbers are given.
\end{abstract}

Keywords Vietoris-Rips complex - Quasi-Vietoris-Rips complex · Betti numbers · Extremal combinatorics

\section{Introduction}

In this paper we consider extremal Betti numbers of Vietoris-Rips complexes. Given a finite set of points $S$ in Euclidean space $\mathbb{R}^{d}$, we define the Vietoris-Rips complex $R^{\delta}(S)$ as the simplicial complex whose faces are given by all subsets of $S$ with diameter at most $\delta$. By scaling, we assume that $\delta=1$ and take $R(S):=R^{1}(S)$. Our main goal in this paper is to determine the maximum Betti numbers of $R(S)$ in terms of $|S|$ and $d$.

Finding such bounds is a natural problem in combinatorial geometry. Several authors, including Björner and Kalai [1], have considered other types of bounds relating to Betti numbers and simplicial complexes. Since every Vietoris-Rips complex is flag, such a complex is also determined by the edges it contains. This adds a flavor of extremal graph theory to our work.

Vietoris-Rips complexes have a wide range of applications. Vietoris [23] used them to calculate the homology groups of metric spaces. Other applications include geometric group theory [11], simplicial approximation of point-cloud data [2-5, 13, 18], and modeling communication between nodes in sensor networks [8, 9, 14]. In the specific case of the Euclidean plane, the topology of Vietoris-Rips complexes is

M. Goff $(\bowtie)$

Department of Mathematics, University of Washington, Seattle, USA

e-mail: pepper20007@yahoo.com 
studied by Chambers, de Silva, Erickson, and Ghrist [6]. Vietoris-Rips complexes are used in manifold reconstruction by Chazal and Oudot [7].

For a fixed base field $\mathbf{k}$, we denote the reduced homology groups of a simplicial complex $\Gamma$ by $\tilde{H}_{p}(\Gamma ; \mathbf{k})$. We use reduced homology to simplify usage of the MayerVietoris sequence. The Betti numbers are given by $\tilde{\beta}_{p}(\Gamma ; \mathbf{k}):=\operatorname{dim}_{\mathbf{k}}\left(\tilde{H}_{p}(\Gamma ; \mathbf{k})\right)$. We define

$$
M_{p, d}(n ; \mathbf{k}):=\max \left\{\tilde{\beta}_{p}(R(S) ; \mathbf{k}): S \subset \mathbb{R}^{d},|S| \leq n\right\} .
$$

The values of $\tilde{\beta}_{p}(\Gamma ; \mathbf{k})$ and $M_{p, d}(n ; \mathbf{k})$ may depend on $\mathbf{k}$. However, all of our results are independent of $\mathbf{k}$, and so $\mathbf{k}$ will be fixed and suppressed from our notation. Our methods break down, though, when the coefficients are not chosen to be a field.

The Betti numbers of random Vietoris-Rips complexes are discussed by Kahle [15]. Under Kahle's set-up, points are chosen from a common distribution on a manifold, and $\tilde{\beta}_{p}(R(S))$ grows at most linearly in the number of vertices. By contrast, our examples of super-linear growth in the Betti numbers arise from specialized constructions. Kahle also explores Betti numbers of general random clique complexes [16]. Both papers make heavy use of methods from probability theory, algebraic topology, and Morse theory.

We review notation and some facts on simplicial complexes and algebraic topology in Sect. 2. One of our most important inductive tools, which we use in the proof of every upper bound result, is that if $\Gamma$ is a simplicial complex, $v$ is a vertex in $\Gamma$, and $\Delta$ is the complex that results from removing $v$ from $\Gamma$, then $\tilde{\beta}_{p}(\Gamma) \leq \tilde{\beta}_{p}(\Delta)+\tilde{\beta}_{p-1}(\operatorname{lk}(v))$. In Sect. 3, we prove that $M_{1, d}(n)$ grows linearly in $n$ for each fixed $d$. The lower bound on $M_{1, d}(n)$ follows by a simple construction, and the upper bound follows by an inductive argument on $n$. The inductive step observes that the number of connected components in the Vietoris-Rips complex of a point set contained in a unit ball cannot exceed the $K_{d}$, the kissing number in $\mathbb{R}^{d}$, and so the upper bound is $M_{1, d}(n) \leq\left(K_{d}-1\right) n$.

The rest of Sect. 3 is devoted to a careful study of the structure of the first homology group of a Vietoris-Rips complex, as this is necessary to apply an inductive argument to the second Betti number. We partition $\mathbb{R}^{d}$ into cubes with side length $\epsilon$, choosing $\epsilon$ small enough so that the diameter of each cube is at most 1 . If $S$ is contained in a sphere of fixed size, then we can choose a basis of cycles for the first homology so that all but a constant number of the cycles are of a convenient form that we call $\epsilon$-simple. Such a cycle is of the form $\left(u, u^{\prime}, v^{\prime}, v\right)$, where $u$ and $u^{\prime}$ are in the same $\epsilon$-cube, and $v$ and $v^{\prime}$ are in the same $\epsilon$-cube. After some additional refinements to the set of generators, we choose a specific edge from each generator, and we call the collection of edges a pseudobasis for the point set $S$. If we choose subsets $U$ and $V$ of $S$ to be intersections of $S$ with $\epsilon$-cubes, then the set of edges in the pseudobasis with vertices in $U$ and $V$ is an induced matching in $R(S)$.

Armed with a combinatorial approximation for the first homology of links of vertices, in Sect. 4 we prove that $M_{2,2}(n)$ grows linearly in $n$. The lower bound also follows by a simple construction. For the upper bound, we let $W$ be a maximally sized intersection of $S$ with an $\epsilon$-cube, and we prove that for some $w \in W$, the size of the pseudobasis for $\operatorname{lk}(w)$ and hence $\tilde{\beta}_{1}(\mathrm{lk}(w))$ are bounded by absolute constants. 
This requires a careful analysis of planar geometry and the combinatorial properties of a pseudobasis. Then induction gives us the upper bound on $\tilde{\beta}_{2}(R(S))$.

Also in Sect. 4, for each fixed $d$ we prove that $M_{2, d}(n)=o\left(n^{2}\right)$. In this setting we lack the tools from planar geometry and rely on the properties of a pseudobasis. We choose $W$ as before, and we show that by restricting the pseudobasis of $1 \mathrm{k}(w)$ for all $w \in W$ to two $\epsilon$-cubes, we get a collection of disjoint induced matchings on a bipartite graph. The proof is finished by citing a deep result on the sizes of disjoint induced matchings. We complete Sect. 4 by giving a construction to prove that $M_{2,5}(n)=\Omega\left(n^{3 / 2}\right)$.

In Sect. 5, we extend the results of the previous sections by showing that for each fixed $p$ and $d, M_{p, d}(n)=o\left(n^{p}\right)$ and $M_{p, 2}(n)=O\left(n^{p-1}\right)$. This follows readily by the inductive argument. We also give constructions to show that $M_{p, 2}(n)=\Omega\left(n^{\lceil p / 2\rceil}\right)$ and $M_{p, 5}(n)=\Omega\left(n^{p / 2+1 / 2}\right)$ for each fixed $p$. Our upper bound on $M_{p, d}(n)$ is better than the trivial upper bound of $\left(\begin{array}{c}n \\ p+1\end{array}\right)$ by slightly more than a factor of $n$.

In Sect. 6, we consider similar bounds on the Betti numbers of related objects known as quasi-Vietoris-Rips complexes. A quasi-Vietoris-Rips complex is a relaxation of a Vietoris-Rips complex; if the distance between vertices $u$ and $v$ is between some fixed $\alpha$ and 1, the edge $u v$ may be included or excluded arbitrarily. In this setting we are forced to rely on the combinatorial properties of a pseudobasis. We prove that for fixed $d$, the upper bound on $\tilde{\beta}_{2}$ is within a constant multiple of the maximum number of edges in $n$ disjoint induced matchings on a bipartite graph with $n$ vertices on each side.

\section{Definitions and Preliminaries}

In this section, we review some basic concepts relating to simplicial complexes, algebraic topology, and matchings in a bipartite graph.

An abstract simplicial complex $\Gamma$ on a finite set $S$, called the vertex set, is a collection of subsets, called faces, of $S$ that is closed under inclusion and contains all singleton subsets. A face with two elements is called an edge. A simplex is a simplicial complex that contains all subsets of the vertex set. For convenience, we often suppress commas and braces when expressing faces of a simplicial complex. We also refer to the vertex set of $\Gamma$ by $\mathcal{V}(\Gamma)$. In this section, $\Gamma$ refers to a general simplicial complex, a class which includes Vietoris-Rips complexes and graphs as special cases, unless otherwise specified.

If $v \in \mathcal{V}(\Gamma)$, then we define the $\operatorname{link} \operatorname{lk}_{\Gamma}(v)$, or $\operatorname{lk}(v)$ when $\Gamma$ is implicit, as the set of faces $F$ in $\Gamma$ such that $F \cup\{v\} \in \Gamma$ and $v \notin F$. The closed star $\operatorname{st}_{\Gamma}(v)=\operatorname{st}(v)$ is the set of faces $F$ in $\Gamma$ such that $F \cup\{v\} \in \Gamma$. For a Vietoris-Rips complex $R(S)$, closed stars and links are also Vietoris-Rips complexes. For a vertex $v$, define the neighbor set $N(v)$ to be the set of vertices $u$ such that $0<\operatorname{dist}(u, v) \leq 1$. Then for $v \in R(S)$, we have that $\operatorname{lk}(v)=R(N(v))$ and $\operatorname{st}(v)=R(N(v) \cup\{v\})$. The induced subcomplex $\Gamma[W]$ for a set of vertices $W$ is the set of faces in $\Gamma$ that are also contained in $W$ (for technical reasons, we allow the possibility that $W \not \subset \mathcal{V}(\Gamma)$ ). For a Vietoris-Rips complex $R(S)$ with $W \subset S$, we have that $R(S)[W]=R(W)$. 
Every Vietoris-Rips complex is a flag complex. A flag complex, also called a clique complex, is a simplicial complex $\Gamma$ such that $F \in \Gamma$ whenever all 2-subsets of $F$ are edges in $\Gamma$. Thus a flag complex is determined by its edges.

We use several basic concepts from algebraic topology; see Hatcher's book [12] for a good overview. For a simplicial complex $\Gamma$, let $\Delta_{p}(\Gamma)$ be the vector space over $\mathbf{k}$ of formal sums of $p$-faces with coefficients in $\mathbf{k}$. For the moment we assume that $\mathcal{V}(\Gamma)$ is given by integers 1 to $|\mathcal{V}(\Gamma)|$. Let $\partial_{p}: \Delta_{p}(\Gamma) \rightarrow \Delta_{p-1}(\Gamma)$ be the linear map that takes $F=\left\{v_{0}, v_{1}, \ldots, v_{p}\right\}$ to $\sum_{j=0}^{p}(-1)^{j}\left(F-\left\{v_{j}\right\}\right)$, assuming that $v_{0}<v_{1}<\cdots<v_{p}$. It is easy to check that $\partial_{p} \circ \partial_{p+1}=0$, and we define

$$
\tilde{H}_{p}(\Gamma ; \mathbf{k})=\tilde{H}_{p}(\Gamma):=\frac{\operatorname{ker} \partial_{p}}{\operatorname{im} \partial_{p+1}} \quad \text { and } \quad \tilde{\beta}_{p}(\Gamma ; \mathbf{k})=\tilde{\beta}_{p}(\Gamma):=\operatorname{dim}_{\mathbf{k}}\left(\tilde{H}_{p}(\Gamma)\right) .
$$

Up to isomorphism, the homology groups are independent of the ordering of the vertices, and so an ordering is not assumed.

We make frequent use of the Mayer-Vietoris sequence, which asserts that for a simplicial complex $W$ that can be written as a union of subcomplexes $U \cup V$, the following sequence is exact:

$$
\cdots \rightarrow \tilde{H}_{p+1}(W) \rightarrow \tilde{H}_{p}(U \cap V) \rightarrow \tilde{H}_{p}(U) \oplus \tilde{H}_{p}(V) \rightarrow \tilde{H}_{p}(W) \rightarrow \cdots
$$

The following application of the Mayer-Vietoris sequence is one of our most important inductive tools.

Lemma 2.1 Let $\Gamma$ be a simplicial complex, and consider $v \in \mathcal{V}(\Gamma)$. Then for all $p$, we have $\tilde{\beta}_{p}(\Gamma) \leq \tilde{\beta}_{p}(\Gamma[\mathcal{V}(\Gamma)-\{v\}])+\tilde{\beta}_{p-1}(\operatorname{lk}(v))$.

Proof Consider $\Delta:=\Gamma[\mathcal{V}(\Gamma)-\{v\}]$ and $\Delta^{\prime}:=\operatorname{st}_{\Gamma}(v)$. Then $\Delta \cup \Delta^{\prime}=\Gamma$ and $\Delta \cap$ $\Delta^{\prime}=\operatorname{lk}(v)$. Since $\Delta^{\prime}$ is a cone-that is, $v$ is contained in all maximal faces of $\Delta^{\prime}$ all of its homology groups vanish. The lemma then follows from the Mayer-Vietoris sequence.

Let $\Gamma$ and $\Delta$ be two simplicial complexes. We define their simplicial join $\Gamma * \Delta$ to have vertex set $\mathcal{V}(\Gamma) \sqcup \mathcal{V}(\Delta)$ and faces $F \cup G$ for all $F \in \Gamma$ and $G \in \Delta$. For $p \geq 0$, the Künneth Formula [12] gives

$$
\tilde{\beta}_{p}(\Gamma * \Delta)=\sum_{i+j=p-1} \tilde{\beta}_{i}(\Gamma) \tilde{\beta}_{j}(\Delta)
$$

noting that $\tilde{\beta}_{-1}(\Gamma)=1$ if $\Gamma=\{\emptyset\}$ and $\tilde{\beta}_{-1}(\Gamma)=0$ otherwise. Let $S$ and $S^{\prime}$ be disjoint subsets of $\mathbb{R}^{d}$ such that $\operatorname{dist}\left(s, s^{\prime}\right) \leq 1$ for all $s \in S$ and $s^{\prime} \in S^{\prime}$. Then $R\left(S \cup S^{\prime}\right)=R(S) * R\left(S^{\prime}\right)$.

Given a simplicial complex $\Gamma$ with some vertices $v_{1}, \ldots, v_{r}$, subscripts mod $r$, and edges $v_{i} v_{i+1}$ for each $i$, the notation $C=\left(v_{1}, \ldots, v_{r}\right)$ refers to the cycle in $\Gamma$. We equivalently think of $C$ as the simplicial 1-chain $\sum_{i=1}^{r} \pm v_{i} v_{i+1}$, with signs chosen so that $\partial C=0$. We denote by $[C]_{\Gamma}$, or $[C]$ when $\Gamma$ is clear from context, the equivalence class of $C$ in $\tilde{H}_{1}(\Gamma)$. 
We make use of complexity notation. Given positive functions $f(n)$ and $g(n)$, the notation $f(n)=O(g(n))$ means that there exists a constant $C$ such that $f(n)<$ $C g(n)$ for sufficiently large $n$. By $f(n)=o(g(n))$, we mean that for every $\epsilon>0$, we have that $f(n)<\epsilon g(n)$ for sufficiently large $n$. The expressions $f(n)=\Omega(g(n))$ and $f(n)=\omega(g(n))$ mean respectively that $g(n)=O(f(n))$ and $g(n)=o(f(n))$. By $f(n)=\theta(g(n))$, we mean that $f(n)=O(g(n))$ and $f(n)=\Omega(g(n))$.

We express some of our bounds in terms of disjoint induced matchings. Let $G$ be a bipartite graph. Then a matching $M$ is a set of edges in $G$ such that no two edges share a common vertex. Furthermore, we say that $M$ is an induced matching if $M$ is also an induced subgraph of $G$. Define $I(n)$ to be the maximum value of $\sum_{M \in \mathcal{M}}|M|$, where $\mathcal{M}$ is a set of disjoint, induced matchings on a bipartite graph with $n$ vertices on each side and $|\mathcal{M}|=n$.

Determining the value of $I(n)$, even to within a multiplicative constant, is a very challenging problem. Via the method of Ruzsa and Szemerédi [21], an example of Elkin [10] can be adapted to show that $I(n)=\Omega\left(n^{2} 2^{-O(\sqrt{\log (n)})}\right)$. As given in the proof of Proposition 10.45 of Tao and Vu [22], $I(n)=O\left(\frac{n^{2}}{\left(\log ^{*}(n)\right)^{1 / 5}}\right)$, where $\log ^{*}(n)$ is the iterated logarithm, the number of natural logarithms one needs to apply to $n$ to obtain a value less than 1 . The $\log ^{*}(n)$ term comes from the usage of the Szemerédi Regularity Lemma in the proof.

For our purposes, the most relevant observations are that $I(n)=\omega\left(n^{3 / 2}\right)$ and $I(n)=o\left(n^{2}\right)$. We also need to quantify the regularity of the growth of $I(n)$. It is easy to see that $I(n)$ is increasing. Furthermore, given a bipartite graph with $n$ vertices on each side and with $n$ disjoint induced matchings, we may create two disjoint copies of the graph. This shows that for all $n, I(2 n) \geq 2 I(n)$ and $I\left(2^{k} n\right) \geq 2^{k} I(n)$, which by monotonicity of $I(n)$ implies that for $n>m$,

$$
I(n) \geq \frac{n}{2 m} I(m) \text {. }
$$

\section{Results on First Homology}

We begin this section by proving the linear growth rate of $M_{1, d}(n)$. The lower bound is approximately $(d-1) n$ for fixed $d$ and large $n$. The upper bound on $\tilde{\beta}_{1}$ is given in terms of the kissing number. The kissing number in $\mathbb{R}^{d}$, which we denote by $K_{d}$, is the maximum number of spheres of radius 1 that are tangent to a central sphere of radius 1 , given that no two spheres overlap in their interiors. See the article of Pfender and Ziegler [20] for some information on kissing numbers. Let $B(v, r)$ be the ball of radius $r$ centered at a point $v$.

Theorem 3.1 Let $k:=\left\lfloor n^{1 / d}\right\rfloor$. Then

$$
d k^{d-1}(k-1)-k^{d}+1 \leq M_{1, d}(n) \leq\left(K_{d}-1\right) n .
$$

Proof First we prove the upper bound. Choose $v \in S$, and let $U$ be a set of vertices that are all in different components of $\operatorname{lk}(v)$. Then $\operatorname{dist}\left(u, u^{\prime}\right)>1$ for all distinct $u$ and $u^{\prime}$ in $U$, which implies that $\angle u v u^{\prime}>\pi / 3$. For all $u \in U$, let $w_{u}$ be the point on 
the ray $\overrightarrow{v u}$ that is distance 2 from $v$. Then all of the $B\left(w_{u}, 1\right)$ are tangent to $B(v, 1)$ and no two of them overlap, which proves that $|U| \leq K_{d}$ and $\tilde{\beta}_{0}(\operatorname{lk}(v)) \leq K_{d}-1$. The upper bound follows by induction on $n$ and Lemma 2.1 .

To establish the lower bound, let $S$ be the set of all points in $\mathbb{R}^{d}$ with integer coordinates between 1 and $k$ inclusive. Then $R(S)$ is connected, and it has $k^{d} \leq n$ vertices, $d k^{d-1}(k-1)$ edges, and no higher faces. It follows that $\tilde{\beta}_{1}(R(S))=$ $d k^{\overline{d-1}}(k-1)-k^{d}+1$ as desired.

The rest of this section is devoted to constructing an approximate combinatorial interpretation of a basis for $\tilde{H}_{1}(R(S))$ called a pseudobasis.

For a given $\epsilon>0$, we partition $\mathbb{R}^{d}$ into $\epsilon$-cubes. An $\epsilon$-cube is a product of halfopen intervals

$$
\left[m_{1} \epsilon,\left(m_{1}+1\right) \epsilon\right) \times \cdots \times\left[m_{d} \epsilon,\left(m_{d}+1\right) \epsilon\right), \quad m_{1}, \ldots, m_{d} \in \mathbb{Z} .
$$

If $\epsilon \leq d^{-1 / 2}$ and $S$ is a finite subset of a single $\epsilon$-cube, then $R(S)$ is a simplex. We define an equivalence relationship $\sim_{\epsilon}$ on vertices and cycles. For $v$ and $v^{\prime}$ in $S$, we say that $v \sim_{\epsilon} v^{\prime}$ if $v$ and $v^{\prime}$ are in the same $\epsilon$-cube. For cycles $C=\left(u_{1}, \ldots, u_{k}\right)$ and $C^{\prime}=\left(v_{1}, \ldots, v_{k}\right)$, we say that $C \sim_{\epsilon} C^{\prime}$ if, perhaps under a cyclic rotation of vertices, each $u_{i} \sim_{\epsilon} v_{i}$. If $C=\left(u, u^{\prime}, v^{\prime}, v\right)$ is a cycle with $u \sim_{\epsilon} u^{\prime}$ and $v \sim_{\epsilon} v^{\prime}$, then we say that $C$ is $\epsilon$-simple.

The bipartite core of a graph $G$ on vertex set $U \sqcup V$ is obtained by deleting all edges of $G[U]$ and $G[V]$, and then deleting all isolated vertices. For sets $U$ and $V$ of vertices in $\mathbb{R}^{d}$, we denote by $G_{S}[U, V]$ the bipartite core of the graph of $R(S)$ on $U \sqcup V$.

For the remainder of the paper, we define the values

$$
\kappa_{d, r, \epsilon}:=(\lceil 2 r / \epsilon\rceil+1)^{d} \quad \text { and } \quad C_{d, r, \epsilon}:=\sum_{i=3}^{2 \kappa_{d, r, \epsilon}}\left(\kappa_{d, r, \epsilon}\right)^{i}
$$

whenever $d, r$, and $\epsilon$ are defined. Suppose that $S$ is a point configuration contained in a ball of radius $r$. Then $S$ is covered by a set of at most $\kappa_{d, r, \epsilon} \epsilon$-cubes. There are at most $\left(\kappa_{d, r, \epsilon}\right)^{i}$ equivalence classes under $\sim_{\epsilon}$ of cycles of length $i$ in $S$, and there are at most $C_{d, r, \epsilon}$ equivalence classes under $\sim_{\epsilon}$ of cycles of length at most $2 \kappa_{d, r, \epsilon}$ in $S$. We define a partitioning of $S$, called $P_{\epsilon} S$, so that each element of $P_{\epsilon} S$ is the intersection of $S$ with an $\epsilon$-cube. We consider $\emptyset$ as an element of $P_{\epsilon} S$.

Definition 3.2 Let $S \subset \mathbb{R}^{d}$ be contained in a ball of radius $r$, and let $W \in P_{\epsilon} S$. A $(W, r, \epsilon)$-pseudobasis of $S$ (equivalently, a pseudobasis for the Vietoris-Rips complex $R(S)$ ) is a set of edges $E$, which we partition into subsets $E_{U, V}=E_{V, U}$ for all distinct $U$ and $V$ in $P_{\epsilon} S$, such that

$$
|E| \geq \tilde{\beta}_{1}(R(S))-C_{d, r, \epsilon}-\left(\begin{array}{c}
\kappa_{d, r, \epsilon} \\
2
\end{array}\right)
$$

and the following conditions are satisfied. 
(PB1) Every edge in $E_{U, V}$ contains a vertex in $U$ and a vertex in $V$.

(PB2) If neither $U$ nor $V$ is $W$, and $u v \in E_{U, V}$, then there is no face $w u v$ for any $w \in W$.

(PB3) No two edges of $E_{U, V}$ are in the same component of $G_{S}[U, V]$.

(PB4) Let $e$ and $f$ be edges in $E_{U, V}$, and let $G_{e}$ and $G_{f}$ be the components of $G_{S}[U, V]$ that contain $e$ and $f$ respectively. Then there is no vertex $s \in S$ such that $\operatorname{lk}(s)$ contains an edge in $G_{e}$ and an edge in $G_{f}$.

If $W$ is a subset of $\mathbb{R}^{d}$ that is not contained in $S$, but $W \cap S$ is in $P_{\epsilon} S$, then by a $(W, r, \epsilon)$-pseudobasis we mean a $(W \cap S, r, \epsilon)$-pseudobasis. An important observation is that PB1 and PB3 imply that $E_{U, V}$ is an induced matching in $G_{S}[U, V]$.

Theorem 3.3 Let $S \subset \mathbb{R}^{d}$ be contained in a ball of radius $r$, let $W \in P_{\epsilon} S$, and suppose that $\epsilon \leq d^{-1 / 2}$. Then $S$ has a $(W, r, \epsilon)$-pseudobasis.

We begin the proof with a lemma on the existence of a set of $\epsilon$-simple cycles that is linearly independent in $\tilde{H}_{1}(R(S))$.

Lemma 3.4 Fix $\epsilon \leq d^{-1 / 2}$, and let $S \subset \mathbb{R}^{d}$ be contained in a ball of radius $r$. Then there exists a set of $\tilde{\beta}_{1}(R(S))-C_{d, r, \epsilon}$ cycles that are $\epsilon$-simple in $R(S)$, and this set is linearly independent in $\tilde{H}_{1}(R(S))$.

Proof It is a standard fact in algebraic topology that $\tilde{H}_{1}(R(S))$ has a basis $\tilde{B}$ such that every element of $\tilde{B}$ is the equivalence class of a cycle. Let $B$ be the corresponding set of cycles. If $[C]$ is the equivalence class of a self-intersecting cycle or a cycle with a chord (that is, an edge between vertices that are not adjacent in the cycle), then $[C]$ can be written as the sum of the equivalence classes of two smaller cycles. Thus by induction $\tilde{H}_{1}(R(S))$ is generated by the equivalence classes of non-self-intersecting, chord-free cycles, and in particular we may assume that every element of $B$ is a nonself-intersecting, chord-free cycle.

In any simplicial complex, if $C$ is a non-self-intersecting, chord-free cycle with at least four vertices, then the subcomplex induced on the vertices of $C$ does not contain two-dimensional faces. If $\left(v_{1}, \ldots, v_{k}\right) \in B$, then no three vertices are in the same $\epsilon$ cube, and $k \leq 2 \kappa_{d, r, \epsilon}$. Thus the elements in $B$ represent at most $C_{d, r, \epsilon}$ equivalence classes under $\sim_{\epsilon}$.

Let $C=\left(u_{1}, \ldots, u_{k}\right)$ and $C^{\prime}=\left(v_{1}, \ldots, v_{k}\right)$ be two cycles in $B$ that are not $\epsilon$ simple and that satisfy $C \sim_{\epsilon} C^{\prime}$. See Fig. 1 for an illustration. There exist edges $u_{i} v_{i}$ for all $i$, and so

$$
\left[C^{\prime}\right]=[C]+\left[v_{1}, v_{2}, u_{2}, u_{1}\right]+\cdots+\left[v_{k-1}, v_{k}, u_{k}, u_{k-1}\right]+\left[v_{k}, v_{1}, u_{1}, u_{k}\right]
$$

The operation of removing $C^{\prime}$ from $B$, adding the cycles $\left[v_{i}, v_{i+1}, u_{i+1}, u_{i}\right]$ for each $i$ and $\left[v_{k}, v_{1}, u_{1}, u_{k}\right]$, and taking a maximal linearly independent subset in $\tilde{H}_{1}(R(S))$ preserves the span of $B$ in $\tilde{H}_{1}(R(S))$. Furthermore, this operation strictly decreases the number of non- $\epsilon$-simple cycles in $B$. By induction, we assume that non- $\epsilon$-simple cycles in $B$ comprise at most one element from each equivalence class under $\sim_{\epsilon}$. 
Fig. 1 The outer cycle is the sum of the inner cycle and six $\epsilon$-simple cycles

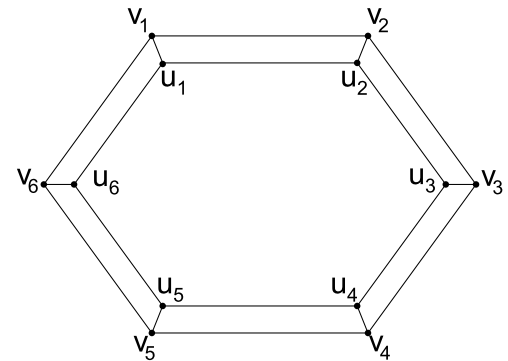

Fig. 2 Rectification: replace $\left(u_{1}, v_{1}, v_{2}, u_{2}\right)$ with $\left(u, v, v_{1}, u_{1}\right)$ and $\left(u, v, v_{2}, u_{2}\right)$

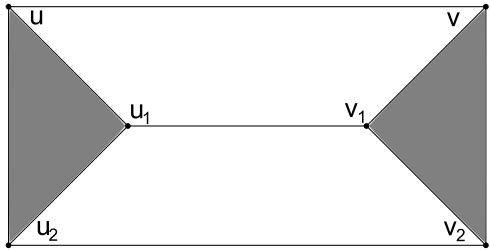

Now remove all non- $\epsilon$-simple cycles from $B$. After this operation, we have that $|B| \geq \tilde{\beta}_{1}(R(S))-C_{d, r, \epsilon}$, and $B$ is linearly independent in $\tilde{H}_{1}(R(S))$.

Proof of Theorem 3.3 We prove the theorem by construction. Let $B$ be a set of $\tilde{\beta}_{1}(R(S))-C_{d, r, \epsilon}$ cycles that are $\epsilon$-simple and linearly independent in $\tilde{H}_{1}(R(S))$. The existence of such $B$ is guaranteed by Lemma 3.4. Partition $B$ into subsets $B_{U, V}=B_{V, U}$ for each $U$ and $V$ in $P_{\epsilon} S$ such that each cycle in $B_{U, V}$ contains vertices of $U$ and $V$. Note that $B_{U, U}=\emptyset$ since if $\left\{u_{1}, u_{2}, u_{3}, u_{4}\right\} \subset U$, then $R\left(u_{1}, u_{2}, u_{3}, u_{4}\right)$ is a simplex and is acyclic. We perform two further standardizations to $B$, and then we verify that by letting $E_{U, V}$ consist of a particular edge from each cycle in $B_{U, V}$, the properties of a pseudobasis are fulfilled.

For our first standardization, we define an operation called rectification on $B_{U, V}$ as follows. The process is illustrated in Fig. 2. Choose points $u \in U$ and $v \in V$ such that the edge $u v$ is in some cycle in $B_{U, V}$. If $\left(u_{1}, v_{1}, v_{2}, u_{2}\right) \in B_{U, V}$, then in $\tilde{H}_{1}(R(S))$, we have $\left[u_{1}, v_{1}, v_{2}, u_{2}\right]=\left[u_{1}, v_{1}, v, v_{2}, u_{2}, u\right]$ by the existence of faces $u u_{1} u_{2}$ and $v v_{1} v_{2}$ in $R(S)$. By the existence of the edge $u v$,

$$
\left[u_{1}, v_{1}, v_{2}, u_{2}\right]=\left[u, v, v_{2}, u_{2}\right]-\left[u, v, v_{1}, u_{1}\right] .
$$

In $B_{U, V}$, replace every cycle of the form $\left(u_{1}, v_{1}, v_{2}, u_{2}\right)$, with $u_{1}$ and $u_{2}$ in $U$ and $v_{1}$ and $v_{2}$ in $V$, with cycles $\left(u, v, v_{2}, u_{2}\right)$ and $\left(u, v, v_{1}, u_{1}\right)$, and then replace $B_{U, V}$ with a maximal subset that is linearly independent in $\tilde{H}_{1}(R(S))$. Rectification does not decrease the span of $B$, nor does it increase the number of edges with one vertex in $U$ and the other in $V$ used in cycles of $B_{U, V}$. An edge $u v$ with $u \in U$ and $v \in V$ that is in every cycle of $B_{U, V}$ is called a pivot. By construction, $B_{U, V}$ has a pivot after it has been rectified. Assume now that each $B_{U, V}$ is rectified.

For our second standardization, which is illustrated in Fig. 3, consider subsets $U$ and $V$ in $P_{\epsilon} S-\{W\}$ with a pivot $u v$ for points $u \in U$ and $v \in V$. A butterfly configuration consists of the following: points $w_{1}$ and $w_{2}$ in $W$; distinct cycles $\left(u, v, v_{1}, u_{1}\right)$ 
Fig. 3 A butterfly configuration. Keep $\left(u, v, v_{1}, u_{1}\right)$, and replace $\left(u, v, v_{2}, u_{2}\right)$ with $\left(u_{1}, w_{1}, w_{2}, u_{2}\right)$ and $\left(v_{2}, w_{2}, w_{1}, v_{1}\right)$

Fig. 4 Verifying Property PB3. The edges $u_{1} v_{1}$ and $u_{2} v_{2}$ are in the same component of $G_{S}[U, V]$. The homology classes $\left[u, v, v_{1}, u_{1}\right]$ and $\left[u, v, v_{2}, u_{2}\right]$ are equal, and thus $u_{1} v_{1}$ and $u_{2} v_{2}$ are not both in $E_{U, V}$
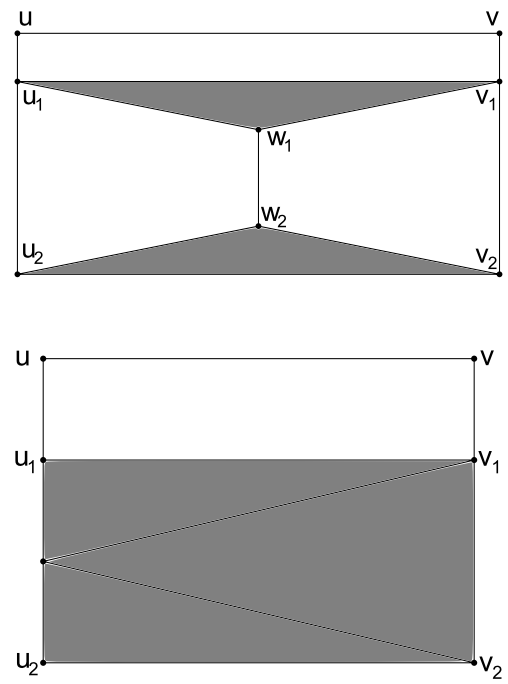

and $\left(u, v, v_{2}, u_{2}\right)$ in $B_{U, V}$; and faces $w_{1} u_{1} v_{1}$ and $w_{2} u_{2} v_{2}$ in $R(S)$. First suppose that $w_{1} \neq w_{2}$. Then in $\tilde{H}_{1}(R(S))$,

$$
\left[u_{1}, v_{1}, v_{2}, u_{2}\right]=\left[u_{1}, w_{1}, v_{1}, v_{2}, w_{2}, u_{2}\right] .
$$

By the existence of an edge $w_{1} w_{2}$, this is equal to $\left[u_{1}, w_{1}, w_{2}, u_{2}\right]+\left[v_{2}, w_{2}, w_{1}, v_{1}\right]$. Combining this with (2),

$$
\left[u, v, v_{2}, u_{2}\right]=\left[u, v, v_{1}, u_{1}\right]+\left[u_{1}, w_{1}, w_{2}, u_{2}\right]+\left[v_{2}, w_{2}, w_{1}, v_{1}\right] .
$$

Consider the operation of removing $\left(u, v, v_{2}, u_{2}\right)$ from $B_{U, V}$; adding $\left(u_{1}, w_{1}, w_{2}, u_{2}\right)$ and $\left(v_{2}, w_{2}, w_{1}, v_{1}\right)$ to $B_{W, U}$ and $B_{W, V}$ respectively; and rectifying $B_{U, V}, B_{W, U}$, and $B_{W, V}$. This operation does not decrease the span of $B$ in $\tilde{H}_{1}(R(S))$, and it strictly decreases the number of edges between $U$ and $V$ that are used in cycles of $B_{U, V}$. In the case that $w_{1}=w_{2}$, we have faces $w_{1} u_{1} u_{2}$ and $w_{1} v_{1} v_{2}$ in $R(S)$ and hence $\left[u_{1}, v_{1}, v_{2}, u_{2}\right]=0$, and by (2), $\left[u, v, v_{1}, u_{1}\right]=\left[u, v, v_{2}, u_{2}\right]$, violating linear independence. By induction on the number of edges between $U$ and $V$ used in cycles of $B_{U, V}$, we assume that no butterfly configurations exist.

Next, for all $U$ and $V$ in $P_{\epsilon} S-\{W\}$ with pivot $u v$ for $u \in U$ and $v \in V$, delete from $B_{U, V}$ every cycle of the form $\left(u, v, v^{\prime}, u^{\prime}\right)$ such that there is a face $w u^{\prime} v^{\prime}$ in $R(S)$ for some $w \in W$. By the non-existence of butterfly configurations, at most one cycle is deleted from $B_{U, V}$, and at most $\left(\begin{array}{c}\kappa_{d, r, \epsilon} \\ 2\end{array}\right)$ cycles are deleted in total.

Now, for all $U$ and $V$ in $P_{\epsilon} S$ with pivot $u v$ for $u \in U$ and $v \in V$, define $E_{U, V}$ to be the set of all $u^{\prime} v^{\prime}$ such that $\left(u, v, v^{\prime}, u^{\prime}\right)$ is a cycle in $B_{U, V}$, and let $E$ be the union of all the $E_{U, V}$. By construction, $|E| \geq \tilde{\beta}_{1}(R(S))-C_{d, r, \epsilon}-\left(\begin{array}{c}\kappa_{d, r, \epsilon} \\ 2\end{array}\right)$, and PB1 and PB2 are fulfilled for $E$.

To verify PB3, we show that for vertices $u_{1}$ and $u_{2}$ in $U$ and $v_{1}$ and $v_{2}$ in $V$ with the edges $u_{1} v_{1}$ and $u_{2} v_{2}$ in the same component of $G_{S}[U, V]$, we cannot have both $u_{1} v_{1}$ and $u_{2} v_{2}$ in $E_{U, V}$, as shown in Fig. 4. By linear independence of $B_{U, V}$, it 
Fig. 5 Verifying Property PB4.

The edges $e:=u_{1} v_{1}$ and $f:=u_{2} v_{2}$ are not both in $E_{U, V}$ with all shown faces present

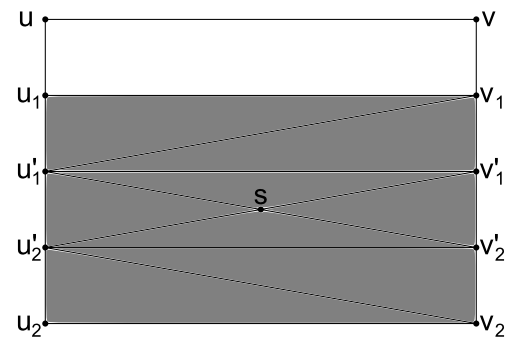

suffices to show that the homology classes $\left[u, v, v_{1}, u_{1}\right]$ and $\left[u, v, v_{2}, u_{2}\right]$ are equal. In the case that $v_{1}=v_{2}$, the claim holds by the existence of faces $u_{1} u_{2} v_{1}$ and $u u_{1} u_{2}$. The claim similarly holds in the case that $u_{1}=u_{2}$, and it holds in general by induction on the length of the shortest path between the edges.

To verify PB4, consider the following configuration: points $s \in S ; u_{1}, u_{2}, u_{1}^{\prime}$, $u_{2}^{\prime} \in U$; and $v_{1}, v_{2}, v_{1}^{\prime}, v_{2}^{\prime} \in V$; edges $e:=u_{1} v_{1}$ and $u_{1}^{\prime} v_{1}^{\prime}$ in the same component of $G_{S}[U, V]$; edges $f:=u_{2} v_{2}$ and $u_{2}^{\prime} v_{2}^{\prime}$ in the same component of $G_{S}[U, V]$; and faces $s u_{1}^{\prime} v_{1}^{\prime}$ and $s u_{2}^{\prime} v_{2}^{\prime}$ in $R(S)$; as shown in Fig. 5. We must show that $e$ and $f$ are not both in $E_{U, V}$, and it suffices to show by linear independence of $B_{U, V}$ that the homology classes $\left[u, v, v_{1}, u_{1}\right]$ and $\left[u, v, v_{2}, u_{2}\right]$ are equal. By (2), it suffices to show that $\left[u_{1}, v_{1}, v_{2}, u_{2}\right]=0$. This is equivalent to $\left[u_{1}^{\prime}, v_{1}^{\prime}, v_{2}^{\prime}, u_{2}^{\prime}\right]=0$ by the argument of the previous paragraph. This holds by the existence of faces $s u_{1}^{\prime} v_{1}^{\prime}, s u_{2}^{\prime} v_{2}^{\prime}, s u_{1}^{\prime} u_{2}^{\prime}$, and $s v_{1}^{\prime} v_{2}^{\prime}$ in $R(S)$.

\section{Results on Second Homology}

In this section, we prove upper bounds on $M_{2,2}(n)$ and $M_{2, d}(n)$ and a lower bound on $M_{2,5}(n)$. Throughout this section, the phrase "the $d$-dimensional hypotheses" is shorthand for the following: $S$ is a subset of $\mathbb{R}^{d}$ of cardinality at most $n, W$ in $P_{\epsilon} S$ is chosen to be of maximum cardinality, $E^{w}$ is a pseudobasis for $1 \mathrm{k}(w)$ for every $w \in W$, and for every $U$ and $V$ in $P_{\epsilon} S$, the set of edges in $E^{w}$ with one vertex in $U$ and the other in $V$ is denoted by $E_{U, V}^{w}$. We also mean that $r:=3 / 2$ and $\epsilon:=d^{-1 / 2}$, except when $d=2$, in which case we take $\epsilon:=0.01$. We begin with $M_{2,2}(n)$.

Theorem 4.1 We have that $M_{2,2}(n)=\Theta(n)$. In particular $M_{2,2}(n) \geq\lfloor n / 2\rfloor-2$.

Proof Take the two-dimensional hypotheses. The lower bound on $M_{2,2}(n)$ is established by a more general construction in the proof of Theorem 5.2.

We claim that for all $U$ and $V$ in $P_{\epsilon} S$,

$$
\sum_{w \in W}\left|E_{U, V}^{w}\right| \leq 10|W|
$$

Observe that $\bigcup_{w \in W} N(w)$ is contained in a disc of radius $r$. Hence there are at most $\left(\begin{array}{c}\kappa_{d, r, \epsilon} \\ 2\end{array}\right)$ pairs $U$ and $V$ in $P_{\epsilon} S$ such that $E_{U, V}^{w}$ is non-empty for some $w \in W$, and so 
Fig. 6 Edges in a pseudobasis for $1 \mathrm{k}(w)$. In this figure,

$E_{U, V}=\left\{u_{1} v_{1}, u_{2} v_{2}, u_{3} v_{3}\right\}$ and $U_{w}=\left\{u_{1}, u_{2}, u_{3}\right\}$

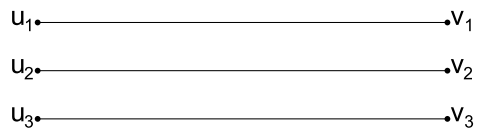

assuming the above claim, it follows that

$$
\sum_{w \in W}\left|E^{w}\right| \leq 10\left(\begin{array}{c}
\kappa_{d, r, \epsilon} \\
2
\end{array}\right)|W|,
$$

and that there exists some $w \in W$ such that $\left|E^{w}\right| \leq 10\left(\begin{array}{c}\kappa_{d, r, \epsilon} \\ 2\end{array}\right)$. By construction of $E^{w}$,

$$
\tilde{\beta}_{1}(\operatorname{lk}(w)) \leq 10\left(\begin{array}{c}
\kappa_{d, r, \epsilon} \\
2
\end{array}\right)+C_{d, r, \epsilon}+\left(\begin{array}{c}
\kappa_{d, r, \epsilon} \\
2
\end{array}\right) .
$$

The theorem follows by Lemma 2.1 and induction on $|S|$.

The proof of the claim is accomplished over the next five lemmas. Lemma 4.2 is need both for the $d=2$ and higher $d$ cases, and it helps us limit the size of $E_{U, W}^{w}$. Lemma 4.3, regarding the arrangement of four points that form two edges in $R(S)$ that intersect in the plane, is found in the work of Chambers et al. [6, Proposition 2.1]. Lemma 4.4 is series of numerical claims about arrangements of points in the plane. We prove the claim for $V=W$ in Lemma 4.5 and for $U \neq W$ and $V \neq W$ in Lemma 4.6.

Lemma 4.2 Take the d-dimensional hypotheses, and choose a subset of points $U$ in $P_{\epsilon} S-\{W\}$. For each $w \in W$, let $U_{w}$ be the set of points that are in $U$ and are also contained in some edge of $E_{U, W}^{w}$, as illustrated in Fig. 6. Consider distinct $w$ and $w^{\prime}$ in $W$ and distinct $a, b$, and $c$ in $U_{w^{\prime}}$. Then not all of $a w, b w, c w$ are edges in $R(S)$. In particular, $\left|U_{w} \cap U_{w^{\prime}}\right| \leq 2$.

Proof By definition of $U_{w^{\prime}}$, there exist distinct vertices $x, y$, and $z$ in $W$ such that $E_{U, W}^{w^{\prime}}$ contains edges $a x, b y$, and $c z$. By $\mathrm{PB} 4, \operatorname{lk}(w)$ contains at most one edge of $E_{U, W}^{w^{\prime}}$, say $a x$ if any of $a x, b y$, or $c z$. If $b w$ is an edge in $R(S)$, then since $1 \mathrm{k}(w)$ contains $b$, we have that $\operatorname{lk}(w)$ does not contain $y$ to insure that $\operatorname{lk}(w)$ does not contain by. But $y$ and $w$ are both in $W$ and $\operatorname{dist}(w, y)<1$, and so it must be that $w=y$. Likewise, if $c w \in R(S)$, then $w=z$. Since $E_{U, W}^{w^{\prime}}$ is a matching, we have that $z \neq y$, and so either $b w$ or $c w$ is not an edge. Hence either $b \notin U_{w}$ or $c \notin U_{w}$. This proves the lemma.

Lemma 4.3 Let $S=\left\{u, u^{\prime}, v, v^{\prime}\right\} \subset \mathbb{R}^{2}$ so that $R(S)$ contains edges $u v$ and $u^{\prime} v^{\prime}$, and suppose that the segments $u v$ and $u^{\prime} v^{\prime}$ intersect. Then $R(S)$ is a cone.

Given $U \subset \mathbb{R}^{2}$, we define a partial ordering on $\mathbb{R}^{2}$ as follows. We say that $v<_{U} v^{\prime}$ if for all $u \in U$, we have $\operatorname{dist}(v, u)<\operatorname{dist}\left(v^{\prime}, u\right)$. For sets of points $U$ and $V$, define

$$
\begin{aligned}
\operatorname{dist}(U, V) & :=\inf \{\operatorname{dist}(u, v): u \in U, v \in V\} \quad \text { and } \\
\operatorname{Dist}(U, V) & :=\sup \{\operatorname{dist}(u, v): u \in U, v \in V\} .
\end{aligned}
$$


Fig. 7 In this configuration, $\theta(\overrightarrow{u v})$ and $\theta(\overrightarrow{\tilde{u}} \tilde{v})$ are within $\pi / 24$ of each other. Hence if $\angle u v v^{\prime}$ is not within $\pi / 24$ of a right angle, then $\angle \tilde{u} \tilde{v} v^{\prime}$ is not a

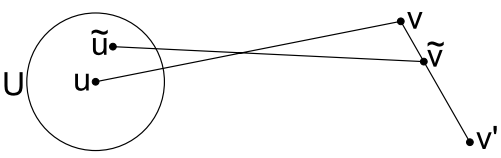
right angle

For a ray $\overrightarrow{u v}$, let $\theta(\overrightarrow{u v})$ denote the angle measured counterclockwise from the positive $x$-axis to $\overrightarrow{u v}$. We take $\theta(\overrightarrow{u v}) \bmod 2 \pi$. Let $\operatorname{conv}(S)$ denote the convex hull of a set $S$.

Lemma 4.4 Set $\epsilon:=0.01$. Then the following claims hold.

(A) Let $u, v, u^{\prime}$, and $v^{\prime}$ be points in $\mathbb{R}^{2}$ with $1-4 \epsilon<\operatorname{dist}(u, v)<1+4 \epsilon$; $\operatorname{dist}\left(u, u^{\prime}\right)<2 \epsilon ;$ and $\operatorname{dist}\left(v, v^{\prime}\right)<2 \epsilon$. Then $\theta\left(\overrightarrow{u^{\prime}} v^{\prime}\right)$ is within $\pi / 24$ of $\theta(\overrightarrow{u v})$.

(B) Let $u, v$, and $v^{\prime}$ be as above, and let $U:=B(u, 2 \epsilon)$. If $v$ and $v^{\prime}$ are incomparable under $<_{U}$, then $\angle u v v^{\prime}$ is within $\pi / 24$ of a right angle.

(C) Let $u, v$, and $w$ be points in $\mathbb{R}^{2}$ with each of $\operatorname{dist}(u, v)$, $\operatorname{dist}(u, w)$, and $\operatorname{dist}(v, w)$ between $1-4 \epsilon$ and $1+4 \epsilon$. Then all of the angles in the triangle uvw are within $\pi / 24$ of $\pi / 3$.

Proof The first claim follows from straightforward computation, and the third claim follows from the law of cosines.

Now consider the second claim. For $\tilde{u} \in U$ and $\tilde{v} \in \operatorname{conv}\left(v, v^{\prime}\right)$, the first claim implies that $\theta(\overrightarrow{u v})$ is within $\pi / 24$ of $\theta(\overrightarrow{\tilde{u}} \tilde{v})$. As illustrated in Fig. 7, if $\angle u v v^{\prime}$ is not within $\pi / 24$ of a right angle, then $\angle \tilde{u} \tilde{v} v^{\prime}$ is never a right angle for any $\tilde{u} \in U$ and $\tilde{v} \in$ $\operatorname{conv}\left(v, v^{\prime}\right)$. By the intermediate value theorem and connectivity of $U \times \operatorname{conv}\left(v, v^{\prime}\right)$, $\angle \tilde{u} \tilde{v} v^{\prime}$ is either always less than $\pi / 2$ or always greater than $\pi / 2$. A particle moving on a straight line from $v$ to $v^{\prime}$ is either always strictly moving toward $U$ or always strictly moving away from $U$. Hence $v$ and $v^{\prime}$ are comparable under $<_{U}$.

Lemma 4.5 Take the two-dimensional hypotheses, and let $U \in P_{\epsilon} S$. Then $\sum_{w \in W}\left|E_{U, W}^{w}\right| \leq 10|W|$.

Proof Recall that $G_{N(w)}[U, W]$ is the bipartite core of $\operatorname{lk}(w)$ on $U \sqcup W$. For some fixed $u^{*} \in U$ and $w^{*} \in W$, we may assume by rotation that $u^{*}$ is directly above $w^{*}$. If $\operatorname{dist}(U, W)>1$, then $\left|E_{U, W}^{w}\right|=0$ for all $w \in W$. If $\operatorname{Dist}(U, W) \leq 1$, then $\left|E_{U, W}^{w}\right| \leq 1$ for all $w \in W$ by PB3 and the observation that $G_{N(w)}[U, W]$ is a complete bipartite graph. Hence we may assume that $\operatorname{dist}(U, W) \leq 1<\operatorname{Dist}(U, W)$ and hence $1-4 \epsilon<$ $\operatorname{dist}(u, w)<1+4 \epsilon$ for all $u \in U$ and $w \in W$.

Let $U_{w}$ be the set of points that are in $U$ and are also contained in some edge of $E_{U, W}^{w}$, and let $W^{\prime}:=\left\{w \in W:\left|E_{U, W}^{w}\right| \geq 5\right\}$. Note that $\left|U_{w}\right|=\left|E_{U, W}^{w}\right|$ since $E_{U, W}^{w}$ is a matching. It suffices to show that $\sum_{w \in W^{\prime}}\left|U_{w}\right| \leq 6|W|$ by

$$
\sum_{w \in W}\left|E_{U, W}^{w}\right|=\sum_{w \in W^{\prime}}\left|U_{w}\right|+\sum_{w \in W-W^{\prime}}\left|U_{w}\right| \leq \sum_{w \in W^{\prime}}\left|U_{w}\right|+4|W| .
$$

As illustrated in Fig. 8, if $w$ and $w^{\prime}$ are in $W$ with $w<U w^{\prime}$, then there is an edge $w u$ in $G_{N\left(w^{\prime}\right)}[U, W]$ for all $u \in U \cap N\left(w^{\prime}\right)$. Hence $G_{N\left(w^{\prime}\right)}[U, W]$ is connected, and 
Fig. 8 In this configuration, $w<_{U} w^{\prime}$. Hence $w^{\prime}$ is not in $W^{\prime}$. The vertices of $W^{\prime}$ are almost arranged on a horizontal line

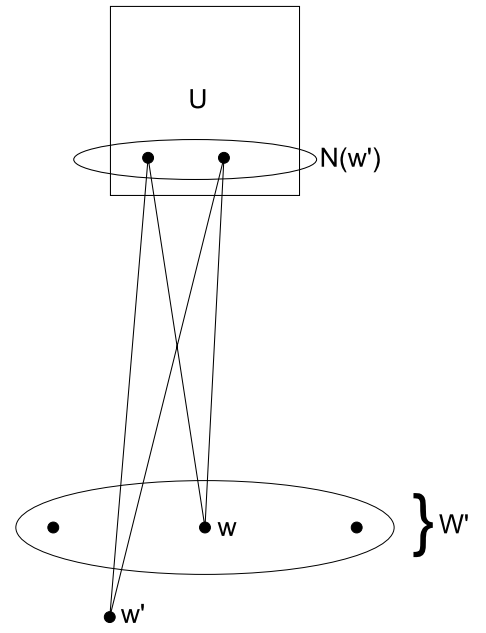

by PB3, $\left|U_{w^{\prime}}\right| \leq 1$. Hence $W^{\prime}$ only contains points of $W$ that are minimal under $<_{U}$. For all distinct $w$ and $w^{\prime}$ in $W^{\prime}$ with $w$ not right of $w^{\prime}$, apply Lemma 4.4(A) to $u^{*}$, $u^{*}, w^{*}$, and $w$ and then Part B to conclude that $\left|\theta\left(\overrightarrow{w w^{\prime}}\right)\right|<\pi / 12$.

Consider $u$ and $u^{\prime}$ in $U$ with $u<_{W} u^{\prime}$ and $w$ and $w^{\prime}$ in $W$. If $w^{\prime} u^{\prime}$ is and edge in $E_{U, W}^{w}$, then $w^{\prime} u$ is an edge in $G_{N(w)}[U, W]$ in the same component as $w^{\prime} u^{\prime}$. Hence we may replace $w^{\prime} u^{\prime}$ with $w^{\prime} u$ and still satisfy all of the pseudobasis properties. Let $U^{\prime}$ be the set of vertices of $U$ that are minimal under $<_{W}$. We may choose $E_{U, W}^{w}$ so that every $U$-vertex of an edge in $E_{U, W}^{w}$ is actually in $U^{\prime}$. As above, for all distinct $u$ and $u^{\prime}$ in $U^{\prime}$ with $u$ not right of $u^{\prime}$, we have that $\left|\theta\left(\overrightarrow{u u^{\prime}}\right)\right|<\pi / 12$. Label the vertices of $U^{\prime}$ by $u_{1}, \ldots, u_{\left|U^{\prime}\right|}$ from left to right.

Consider $w$ and $w^{\prime}$ in $W^{\prime}$ with $w$ left of $w^{\prime}$, and we claim that there cannot exist $a, b, c \in U_{w^{\prime}}$ and $d, e, f \in U_{w}$ with each of $\{a, b, c\}$ left of each of $\{d, e, f\}$, as in Fig. 9. Suppose by way of contradiction that such $a, \ldots, f$ exist. By Lemma 4.2, we may assume without loss of generality that $c w$ and $f w^{\prime}$ are not edges in $R(S)$. By Lemma 4.4(A), both $\theta\left(\overrightarrow{w^{\prime}} c\right)$ and $\theta(\overrightarrow{w f})$ are between $\pi / 2-\pi / 24$ and $\pi / 2+\pi / 24$. Also, as shown above, $|\theta(\overrightarrow{c f})|<\pi / 12$ and $\left|\theta\left(\overrightarrow{w w^{\prime}}\right)\right|<\pi / 12$. By consideration of angles, $w$ and $f$ are on opposite sides of the line $w^{\prime} c$, and $w^{\prime}$ and $c$ are on opposite sides of the line $w f$. Hence the segments $w f$ and $w^{\prime} c$ intersect, and the set $\left\{w, w^{\prime}, c, f\right\}$ violates Lemma 4.3. Thus there cannot exist such $a, \ldots, f$.

For $w \in W^{\prime}$, let $l_{3}(w)$ and $r_{3}(w)$ denote the indices of the points of $U_{w}$ with third smallest and third largest $x$-coordinates respectively. By the previous paragraph, if $w$ and $w^{\prime}$ are points in $W^{\prime}$ with $w$ left of $w^{\prime}$, then

$$
l_{3}\left(w^{\prime}\right) \geq r_{3}(w) \geq l_{3}(w)+\left|U_{w}\right|-5 .
$$

If $W^{\prime}=\left\{w_{1}, \ldots, w_{\left|W^{\prime}\right|}\right\}$ from left to right and $x:=x_{\left|W^{\prime}\right|}$, then by (4) and induction on $k$ we have that $l_{3}\left(w_{k}\right) \geq l_{3}\left(w_{1}\right)+\sum_{i=1}^{k-1}\left(\left|U_{w_{i}}\right|+5\right)$. Hence

$$
l_{3}\left(w_{1}\right)+\sum_{w \in W^{\prime}-\{x\}}\left(\left|U_{w}\right|-5\right) \leq l_{3}(x) \leq\left|U^{\prime}\right|-\left|U_{x}\right|+3 .
$$


Fig. 9 We cannot have a point configuration

$\left\{a, b, c, d, e, f, w, w^{\prime}\right\}$ as above. Dotted lines indicate edges that are not in $R(S)$

Fig. 10 The relative positions of $U, V$, and $W$ and representative points $u^{*}, v^{*}$, and $w^{*}$. We assume that $\theta\left(u^{*} w^{*}\right)$ is $\pi / 6$, and it follows that $\theta\left(v^{*} \vec{w}^{*}\right)$ is approximately $-\pi / 6$
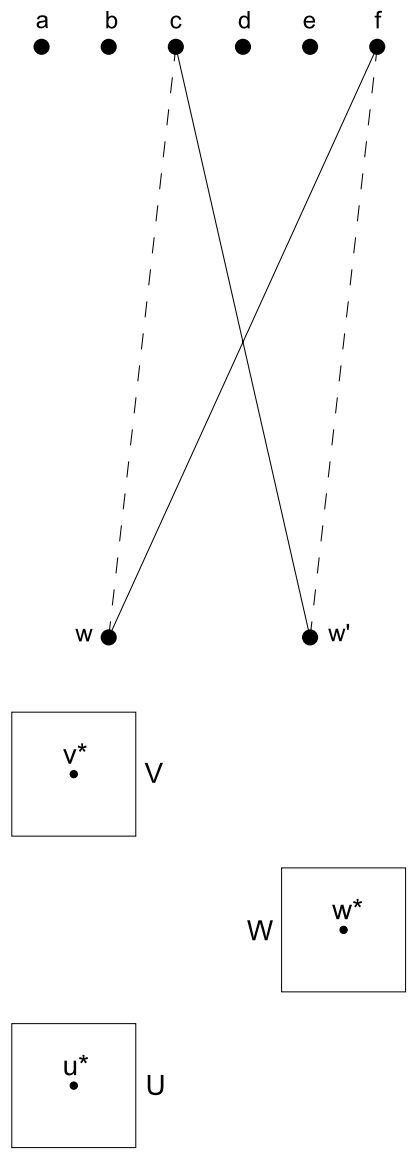

Then

$$
\sum_{w \in W^{\prime}}|U(w)| \leq\left|U^{\prime}\right|+3+5\left(\left|W^{\prime}\right|-1\right)<6|W| .
$$

The latter inequality follows from the assumption that $|W| \geq|U|$. This proves the lemma.

Lemma 4.6 Take the two-dimensional hypotheses, and consider $U$ and $V$ in $P_{\epsilon} S-\{W\}$. Then $\sum_{w \in W}\left|E_{U, V}^{w}\right| \leq 2|W|$.

Proof Our first step is to justify an assumption that the sets $U, V$, and $W$ approximately form an equilateral triangle, as shown in Fig. 10. As above, $G_{N(w)}[U, V]$ is the bipartite core of $\operatorname{lk}(w)$ on $U \sqcup V$. If $\operatorname{dist}(U, V)>1$; or if $\operatorname{dist}(U, W)>1$; or if $\operatorname{dist}(V, W)>1$, then $\left|E_{U, V}^{w}\right|=0$ for all $w \in W$, and the lemma holds. If $\operatorname{Dist}(U, V) \leq 1$, then $G_{N(w)}[U, V]$ is a complete bipartite graph, and hence by PB3, $\left|E_{U, V}^{w}\right| \leq 1$ for all $w \in W$. The claim follows. 
Fig. 11 The arrangement of $W_{u}$ and $v_{u}$

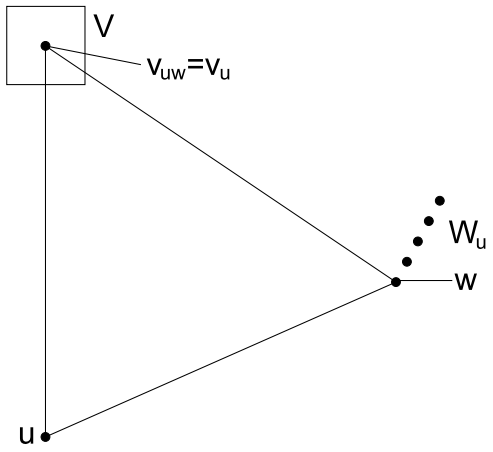

Define $U_{w}$ and $V_{w}$ to be the sets of points that are in $U$ and $V$ respectively and are also contained in edges in $E_{U, V}^{w}$. If $\operatorname{Dist}(U, W) \leq 1$, then consider distinct $w$ and $w^{\prime}$ in $W$, together with an edge $u v$ in $E_{U, V}^{w}$ for points $u \in U_{w}$ and $v \in V_{w}$. By PB2 for $E_{U, V}^{w}, u v$ is not an edge in $1 \mathrm{k}\left(w^{\prime}\right)$, which by $u \in N\left(w^{\prime}\right)$ implies that $v \notin$ $N\left(w^{\prime}\right)$. It follows that $V_{w} \cap V_{w^{\prime}}=\emptyset$. Since each $E_{U, V}^{w}$ is a matching, $\sum_{w \in W}\left|E_{U, V}^{w}\right| \leq$ $|V| \leq|W|$, proving the lemma. Likewise, if $\operatorname{Dist}(V, W) \leq 1$, then the lemma holds.

We assume that

$$
\begin{aligned}
& 1-4 \epsilon<\operatorname{dist}(U, V), \operatorname{dist}(U, W), \operatorname{dist}(V, W) \text { and } \\
& \operatorname{Dist}(U, V), \operatorname{Dist}(U, W), \operatorname{Dist}(V, W)<1+4 \epsilon .
\end{aligned}
$$

Fix points $u^{*} \in U, v^{*} \in V$, and $w^{*} \in W$, and orient the plane so that $\theta\left(u^{*} w^{*}\right)=\pi / 6$ and $u^{*}, v^{*}$, and $w^{*}$ are arranged clockwise in the triangle $u^{*} v^{*} w^{*}$. By Lemma 4.4(C),

$$
\left|\theta\left(v^{*} w^{*}\right)+\pi / 6\right|<\pi / 24 .
$$

For $u \in U$ and $v \in V$, define $W_{u}$ to be the set of points $w$ in $W$ such that some edge of $E_{U, V}^{w}$ contains $u$, as shown in Fig. 11. Analogously, define $W_{v}$ to be the set of points $w$ in $W$ such that some edge of $E_{U, V}^{w}$ contains $v$. For $w \in W_{u}$, define the vertex $v_{u w}$ so that $E_{U, V}^{w}$ contains the edge $u v_{u w}$. We may think of $v_{u w}$ as the "mate" of $u$ in $E_{U, V}^{w}$. Consider distinct $w$ and $w^{\prime}$ in $W_{u}$ with $w^{\prime} \nless_{V} w$. By Lemma 4.4(A) and (5), we have that $\left|\theta\left(v^{*} w\right)+\pi / 6\right|<\pi / 12$, which by Part B implies that either

$$
\left|\theta\left(\vec{w} w^{\prime}\right)-\pi / 3\right|<\pi / 8
$$

or $w<_{V} w^{\prime}$. But if $w<_{V} w^{\prime}$, then $u$ and $v_{u w^{\prime}}$ are both in $N(w)$, and thus $u v_{u w^{\prime}}$ is an edge in $1 \mathrm{k}(w)$ and in $E_{U, V}^{w^{\prime}}$, a contradiction to PB2. We conclude that (6) holds. By Lemma 4.4(A), we have that $\left|\theta\left(u^{*} w\right)-\pi / 6\right|<\pi / 24$, and so by Part $\mathrm{B}, w$ and $w^{\prime}$ are comparable under $<_{U}$. Thus $W_{u}$ is totally ordered under $<_{U}$ and is antichain (that is, no two elements are comparable) under $<_{V}$. Similarly, $W_{v}$ is totally ordered under $<_{V}$ and is an antichain under $<_{U}$.

For all $u \in U$ with $W_{u}$ nonempty, there exists $w \in W_{u}$ that is of maximal distance from $u$; given this $w$, define $v_{u}:=v_{u w}$, as shown in Fig. 11. For all $v \in V$ 
Fig. 12 The arrangement of $w, u, v, w_{u}, w_{v}$

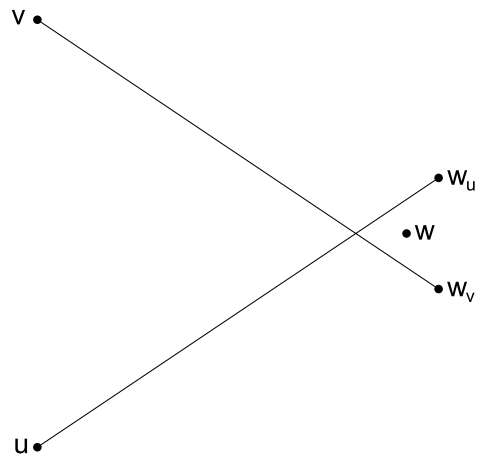

with $W_{v} \neq \emptyset$, define $u_{v}$ similarly. There are at most $|U|$ (resp. $|V|$ ) edges $u v$ in $\bigcup_{w \in W} E_{U, V}^{w}$ such that $v=v_{u}\left(\right.$ resp. $\left.u=u_{v}\right)$.

Suppose by way of contradiction that $\sum_{w \in W}\left|E_{U, V}^{w}\right|>2|W|$. By choice of $W$, $\sum_{w \in W}\left|E_{U, V}^{w}\right|>|U|+|V|$. The $E_{U, V}^{w}$ are disjoint by PB2, and so there exist points $w \in W, u \in U$, and $v \in V$ such that $u v \in E_{U, V}^{w}, u \neq u(v)$, and $v \neq v(u)$. The arrangement of points is illustrated in Fig. 12. Choose points $w_{u} \in W_{u}$ and $w_{v} \in W_{v}$ such that $\operatorname{dist}\left(u, w_{u}\right)>\operatorname{dist}(u, w)$ and $\operatorname{dist}\left(v, w_{v}\right)>\operatorname{dist}(v, w)$. Such $w_{u}$ and $w_{v}$ exist by choice of $w$. By Lemma 4.4(A),

$$
|\theta(\overrightarrow{u w})-\pi / 6|<\pi / 24 .
$$

Combining Part A with (5),

$$
|\theta(v \vec{w})+\pi / 6|<\pi / 12 .
$$

By (6),

$$
\left|\theta\left(w \vec{w}_{u}\right)-\pi / 3\right|<\pi / 8 .
$$

Since $W_{v}$ is an antichain under $<_{U}$, by (7) and Lemma 4.4(B) we have that

$$
\left|\theta\left(w \vec{w}_{v}\right)+\pi / 3\right|<\pi / 12 .
$$

By (7) and (9), $w$ is below the line $u w_{u}$, which by (10) implies that $w_{v}$ is below the line $u w_{u}$. Also, $v$ is above the line $u w_{u}$. By (8) and (10), $w$ is above the line $v w_{v}$, which by (9) implies that $w_{u}$ is above the line $v w_{w}$. Also, $u$ is below the line $v w_{v}$. Hence the segments $u w_{u}$ and $v w_{v}$ intersect. By Lemma 4.3, either $u w_{v}$ or $v w_{u}$ is an edge, yielding either the face $u v w_{v}$ or $u v w_{u}$ by the existence of edges $u v, v w_{v}$, and $u w_{u}$. This contradicts PB2 for $E^{w}$. We conclude that $\sum_{w \in W}\left|E_{U, V}^{w}\right| \leq 2|W|$ as desired.

Specifically, it follows by the inductive argument and (3) that

$$
M_{2,2}(n) \leq\left(11\left(\begin{array}{c}
\kappa_{2,1.5,0.01} \\
2
\end{array}\right)+C_{2,1.5,0.01}\right) n .
$$


The coefficient is quite large, and we expect that a linear upper bound with a modest coefficient should exist.

We now turn our attention to higher dimensions. Lacking the tools from plane geometry, the proof of the following theorem relies on the pseudobasis properties. Recall that $I(n)$ is the maximum size of the union of $n$ disjoint matchings on a bipartite graph with $n$ vertices on each side.

Theorem 4.7 For all fixed $d, M_{2, d}(n)=O(I(n))$, and thus $M_{2, d}(n)=o\left(n^{2}\right)$.

Proof Take the $d$-dimensional hypotheses. We use some of the same methods as in the proof of Theorem 4.1. Let $S \subset \mathbb{R}^{d}$ with $|S| \leq n$. We have that $\bigcup_{w \in W} N(w)$ is contained in a ball of radius $r$.

We claim that for all $U$ and $V$ in $P_{\epsilon} S$,

$$
\sum_{w \in W}\left|E_{U, V}^{w}\right| \leq|W| \max \left\{\frac{2 I(n)}{n}, 2 \sqrt{n}\right\}=|W| O(I(n) / n) .
$$

Since there are at most $\left(\begin{array}{c}\kappa_{d, r, \epsilon} \\ 2\end{array}\right)$ pairs $\{U, V\} \subset P_{\epsilon} S$ such that $E_{U, V}^{w}$ is non-empty, it then follows that $\sum_{w \in W}\left|E^{w}\right|=|W| O(I(n) / n)$, and that there exists some $w \in W$ such that $\left|E^{w}\right|=O(I(n) / n)$. By construction of $E^{w}, \tilde{\beta}_{1}(\operatorname{lk}(w))=O(I(n) / n)$. Then by Lemma 2.1, $\tilde{\beta}_{2}(R(S))<\tilde{\beta}_{2}(R(S-\{w\}))+O(I(n) / n)$, and the theorem follows by induction on $n$ and (1).

To prove the claim, first consider the case that $V=W$. Let $G$ be the bipartite graph with vertex set identified with $U \sqcup W$ and an edge $u w$ whenever $u$ is a vertex of some edge of $E_{U, W}^{w}$. Then $G$ is a bipartite graph with at most $|W|$ vertices on each side, and by Lemma 4.2, no two vertices in $W$ have three common neighbors in $G$. It follows from a special case of the theorem of Kövári, Sós, and Turán [17] that $G$ has at most $\sqrt{2}(|W|-1)|W|^{1 / 2}+|W|<2|W|^{3 / 2}$ edges. Also, since $E_{U, W}$ is a matching, $G$ has $\sum_{w \in W}\left|E_{U, W}^{w}\right|$ edges. Hence

$$
\sum_{w \in W}\left|E_{U, W}^{w}\right| \leq 2|W|^{3 / 2} \leq 2 n^{1 / 2}|W|
$$

Now consider $U$ and $V$ in $P_{\epsilon} S-\{W\}$. PB1 and PB3 imply that $E_{U, V}^{w}$ is an induced matching in $G_{S}[U, V]$ for all $w \in W$. PB2 implies that $E_{U, V}^{w}$ and $E_{U, V}^{w^{\prime}}$ are disjoint for all $w \neq w^{\prime}$. It follows that

$$
\sum_{w \in W}\left|E_{U, V}^{w}\right| \leq I(|W|) \leq|W| 2 I(n) / n
$$

The first inequality follows from the assumption that $|W| \geq|U|$ and $|W| \geq|V|$, and the second inequality follows from (1).

By construction of a pseudobasis, we have a specific bound of

$$
M_{2, d}(n) \leq n\left(\left(\begin{array}{c}
\kappa_{d, r, \epsilon} \\
2
\end{array}\right) \max \left\{2 n^{1 / 2}, 2 I(n) / n\right\}+C_{d, r, \epsilon}+\left(\begin{array}{c}
\kappa_{d, r, \epsilon} \\
2
\end{array}\right)\right)
$$


with $r=3 / 2$ and $\epsilon=d^{-1 / 2}$. For large $n$,

$$
M_{2, d}(n) \leq 2\left(\begin{array}{c}
\kappa_{d, r, \epsilon} \\
2
\end{array}\right) I(n)+\left(C_{d, r, \epsilon}+\left(\begin{array}{c}
\kappa_{d, r, \epsilon} \\
2
\end{array}\right)\right) n .
$$

We conclude this section with a lower bound on $M_{2,5}(n)$. A $(U, V, Y)$-concentrated simplicial complex, of which the following construction is an example, is a flag complex on vertex set $U \sqcup V \sqcup Y$ with each of $\Gamma[U], \Gamma[V]$, and $\Gamma[Y]$ a simplex.

Theorem 4.8 We have $M_{2,5}(n) \geq\left\lfloor\sqrt{\frac{n}{3}}\right\rfloor^{3}-n \approx(n / 3)^{3 / 2}$.

Proof We prove the result by construction. Set $k:=\left\lfloor\sqrt{\frac{n}{3}}\right\rfloor$. Let $U:=\left\{u_{i, l}, 1 \leq i, l \leq\right.$ $k\} ; V:=\left\{v_{j, l}, 1 \leq j, l \leq k\right\} ;$ and $Y:=\left\{y_{i, j}, 1 \leq i, j \leq k\right\}$ with

$$
\begin{aligned}
& u_{i, l}:=\left(\frac{\sqrt{2}}{2} \cos \left(\frac{i}{n}\right), \frac{\sqrt{2}}{2} \sin \left(\frac{i}{n}\right), 0,0, \frac{l}{n^{3}}\right), \\
& v_{j, l}:=\left(0,0, \frac{\sqrt{2}}{2} \cos \left(\frac{j}{n}\right), \frac{\sqrt{2}}{2} \sin \left(\frac{j}{n}\right), \frac{l}{n^{3}}\right), \text { and } \\
& y_{i, j}:=\left(\frac{\sqrt{2}}{4} \cos \left(\frac{i}{n}\right), \frac{\sqrt{2}}{4} \sin \left(\frac{i}{n}\right), \frac{\sqrt{2}}{4} \cos \left(\frac{j}{n}\right), \frac{\sqrt{2}}{4} \sin \left(\frac{j}{n}\right), \frac{\sqrt{3}}{2}\right) .
\end{aligned}
$$

The subgraphs of $R(S)$ induced on $U, V$, and $Y$ are cliques. The edge set of $R(S)$ contains exactly the following additional edges:

(1) $u_{i, l} v_{j, l}$ for all $1 \leq i, j, l \leq k$,

(2) $u_{i, l} y_{i, j}$ for all $1 \leq i, j, l \leq k$,

(3) $v_{j, l} y_{i, j}$ for all $1 \leq i, j, l \leq k$.

For each $y=y_{i, j} \in Y$, we have that $G_{N(y)}[U, V]$ is an induced matching with edge set $\left\{u_{i, l} v_{j, l}: 1 \leq l \leq k\right\}$. These matchings are disjoint over all $y \in Y$. By Lemma 4.9, $\beta_{2}(R(S)) \geq k^{3}-3 k^{2} \geq\left\lfloor\sqrt{\frac{n}{3}}\right\rfloor^{3}-n$.

Lemma 4.9 Let $\Gamma$ be a $(U, V, Y)$-concentrated simplicial complex. Suppose that for every $y \in Y$, the restriction of $\operatorname{lk}_{\Gamma}(y)$ to the bipartite core of $U \sqcup V$ is an induced matching on $k_{y}$ pairs of vertices, and also that these matchings are disjoint. Then

$$
\tilde{\beta}_{2}(\Gamma) \geq \sum_{y \in Y} k_{y}-|U|-2|Y| .
$$

Proof We first show that for every $u \in U, v \in V$, and $y \in Y$ such that $u v y$ is a face of $\Gamma$, in fact $u v y$ is a maximal face. To see this, consider $u^{\prime} \in U, v^{\prime} \in V$, and $y^{\prime} \in Y$ that are distinct from $u, v$, and $y$. By the assumption that the matchings are disjoint, $u v \notin \operatorname{lk}\left(y^{\prime}\right)$. If $u^{\prime} v y$ (resp. $u v^{\prime} y$ ) is a face, then $u^{\prime} v \in \operatorname{lk}(y)$ (resp. $u v^{\prime} \in \operatorname{lk}(y)$ ), contradicting the assumption that the matchings are induced. Hence none of $u v y u^{\prime}, u v y v^{\prime}$, or $u v y y^{\prime}$ are faces, which implies that $u v y$ is maximal. 
Construct $\Delta$ from $\Gamma$ by removing all faces of the form $u v y$ for $u \in U, v \in V$, and $y \in Y$. Note that $\Delta$ is not flag, but $\Delta[U, V], \Delta[U, Y]$, and $\Delta[V, Y]$ are all flag and $\Delta[U], \Delta[V]$, and $\Delta[Y]$ are all simplices.

We first place an upper bound on $\tilde{\beta}_{1}(\Delta)$. Note that $\Delta[V]$ is a simplex and $\tilde{\beta}_{1}(\Delta[V])=0$. Consider some $U^{\prime} \subset U$ and some $u \in U^{\prime}$. Then $\Lambda:=\operatorname{lk}_{\Delta\left[U^{\prime}, V\right]}(u)$ has at most two components since $\Lambda[U]$ and $\Lambda[V]$ are simplices. Thus $\tilde{\beta}_{0}(\Lambda) \leq 1$. By Lemma 2.1 and induction on $\left|U^{\prime}\right|$, we have that $\tilde{\beta}_{1}\left(\Delta\left[U^{\prime}, V\right]\right) \leq\left|U^{\prime}\right|$ and thus $\tilde{\beta}_{1}(\Delta[U, V]) \leq|U|$. Now consider some $Y^{\prime} \subset Y$ and some $y \in Y^{\prime}$. Then $\Lambda:=$ $\mathrm{lk}_{\Delta\left[Y^{\prime}, U, V\right]}(y)$ has at most three components since $\Lambda[U], \Lambda[V]$, and $\Lambda[Y]$ are simplices. Thus $\tilde{\beta}_{0}(\Lambda) \leq 2$. By Lemma 2.1 and induction on $\left|Y^{\prime}\right|$, we have that $\tilde{\beta}_{1}\left(\Delta\left[Y^{\prime}, U, V\right]\right) \leq|U|+2\left|Y^{\prime}\right|$ and $\tilde{\beta}_{1}(\Delta) \leq|U|+2|Y|$.

The operation of adding a two-dimensional face to a simplicial complex either decreases $\tilde{\beta}_{1}$ by 1 or increases $\tilde{\beta}_{2}$ by 1 . Since $\Gamma$ is constructed from $\Delta$ by adding $\sum_{y \in Y} k_{y}$ two-dimensional faces, $\tilde{\beta}_{2}(\Gamma) \geq \sum_{y \in Y} k_{y}-|U|-2|Y|$.

Label the construction of Theorem 4.8 as $S^{2}(n)$. All points of $U, V$, and $Y$ are within distance $o(1)$ of $(\sqrt{2} / 2,0,0,0,0),(0,0, \sqrt{2} / 2,0,0)$, and $(\sqrt{2} / 4,0, \sqrt{2} / 4,0$, $\sqrt{3} / 2)$ respectively.

\section{Results on Higher Homology}

The results of the previous section can be extended to higher Betti numbers.

Theorem 5.1 Fix $p \geq 2$ and $d$. Then $M_{p, d}(n)=O\left(n^{p-2} I(n)\right)$. Also, $M_{p, 2}(n)=$ $O\left(n^{p-1}\right)$.

Proof We claim that $M_{p, d}(n) \leq n M_{p-1, d}(n)$. To see this, consider $S \subset \mathbb{R}^{d}$ and a point $v \in S$. Then $\operatorname{lk}(v)=R(N(v))$, and so $\tilde{\beta}_{p-1}(\operatorname{lk}(v)) \leq M_{p-1, d}(n)$. By Lemma 2.1, we have that $M_{p, d}(n) \leq M_{p, d}(n-1)+M_{p-1, d}(n)$. The claim follows by induction on $n$ and the observation that $M_{p-1, d}(n)$ is non-decreasing in $n$.

The two statements in the theorem then follow by induction on $p$ and Theorems 4.7 and 4.1 respectively.

Theorem 5.2 For fixed $p>0$, we have that $M_{p, 2}(n)=\Omega\left(n^{\lceil p / 2\rceil}\right)$.

Proof First consider the case that $p=2 k-1$. We prove the result by giving $S \subset \mathbb{R}^{2}$ with $|S| \leq n$ and $\tilde{\beta}_{2 k-1}(R(S)) \geq\left(\left\lfloor\frac{n}{2 k}\right\rfloor-1\right)^{k}$. See Fig. 13 for an illustration. For $0 \leq i \leq k-1$, define

$$
\begin{aligned}
S_{i}^{-} & :=\left\{\left[\begin{array}{cc}
\cos (i / n) & -\sin (i / n) \\
\sin (i / n) & \cos (i / n)
\end{array}\right]\left[\begin{array}{c}
-1 / 2 \\
j n^{-4}
\end{array}\right]: 0 \leq j<\left\lfloor\frac{n}{2 k}\right\rfloor\right\}, \\
S_{i}^{+} & :=\left\{\left[\begin{array}{cc}
\cos (i / n) & -\sin (i / n) \\
\sin (i / n) & \cos (i / n)
\end{array}\right]\left[\begin{array}{c}
1 / 2 \\
j n^{-4}
\end{array}\right]: 0 \leq j<\left\lfloor\frac{n}{2 k}\right\rfloor\right\},
\end{aligned}
$$


Fig. 13 Left: a point configuration in $\mathbb{R}^{2}$ with $\tilde{\beta}_{3}$ large. Right: $\tilde{\beta}_{4}$ is large. Edges between $S_{0}$ and $S_{1}$ are not shown

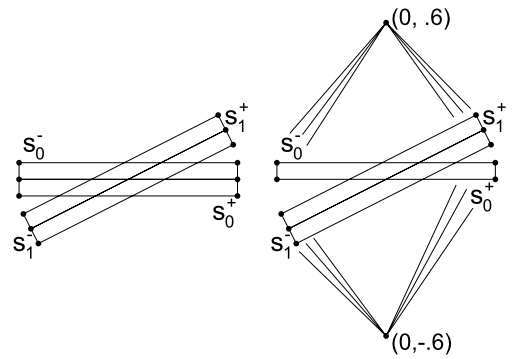

and $S_{i}:=S_{i}^{-} \cup S_{i}^{+}$. In words, $S_{i}$ consists of $\lfloor n / 2 k\rfloor$ pairs of opposing points concentrated on two parallel segments separated by distance 1 . Each $S_{i}$ is a slight counterclockwise rotation of $S_{i-1}$. Each $R\left(S_{i}^{+}\right)$and $R\left(S_{i}^{-}\right)$is a simplex, and the bipartite core of the graph of $R\left(S_{i}^{+} \sqcup S_{i}^{-}\right)$is a matching on all of the vertices. It is easy to check that $\tilde{\beta}_{p}\left(R\left(S_{i}\right)\right)=0$ for $p \neq 1$ and that

$$
\tilde{\beta}_{1}\left(R\left(S_{i}\right)\right)=\left\lfloor\frac{n}{2 k}\right\rfloor-1 .
$$

For all $u \in S_{i}$ and $v \in S_{j}$ with $i \neq j$, we have that $\operatorname{dist}(u, v)<1$. Set $S:=S_{0} \cup$ $\cdots \cup S_{k-1}$, and then $R(S)=R\left(S_{0}\right) * \cdots * R\left(S_{k-1}\right)$. By the Künneth Formula,

$$
\tilde{\beta}_{2 k-1}(R(S))=\left(\left\lfloor\frac{n}{2 k}\right\rfloor-1\right)^{k} .
$$

Now we consider the case that $p=2 k$. Replace the points $(-1 / 2,0)$ and $(1 / 2,0)$ in the above construction with $a:=(0, .6)$ and $b:=(0,-.6)$. See Fig. 13 for an illustration. Then $\tilde{\beta}_{0}(R(a, b))=1$, and all other homology groups of $R(a, b)$ vanish. For all $u \in\{a, b\}$ and $v \in S-\{a, b\}$, we have that $\operatorname{dist}(u, v)<1$. Then

$$
R(S)=R\left(S_{0}-\left\{\left(\frac{1}{2}, 0\right),\left(-\frac{1}{2}, 0\right)\right\}\right) * R\left(S_{1}\right) * \cdots * R\left(S_{k-1}\right) * R(a, b) .
$$

It similarly follows from the Künneth Formula that

$$
\tilde{\beta}_{2 k}(R(S))=\left(\left\lfloor\frac{n}{2 k}\right\rfloor-2\right)\left(\left\lfloor\frac{n}{2 k}\right\rfloor-1\right)^{k-1} .
$$

If $p$ is odd, then label the above construction as $S^{p}(n)$ with $S^{-1}(n):=\emptyset . \mathrm{Ob}$ serve that for odd $p$, all points of $S^{p}(n)$ are within distance $o(1)$ of either $(1 / 2,0)$ or $(-1 / 2,0)$.

Theorem 5.3 For every $p>0$, we have that $M_{p, 5}(n)=\Omega\left(n^{p / 2+1 / 2}\right)$.

Proof The theorem follows for odd $p$ by Theorem 5.2, and so we consider even $p$. We may assume that $n$ is even and define $m:=n / 2$. Let $S:=S^{2}(m)$ as at the end of Sect. 4, and let $S^{\prime}$ be the image of $S^{p-3}(m)$ under the isometry that 
sends $(x, y)$ to $(\sqrt{2} / 4, x, \sqrt{2} / 4, y, \sqrt{3} / 6)$. Every point in $S$ is within distance $o(1)$ of either $(0,0, \sqrt{2} / 2,0,0),(\sqrt{2} / 2,0,0,0,0)$, or $(\sqrt{2} / 4,0, \sqrt{2} / 4,0, \sqrt{3} / 2)$, and every point of $S^{\prime}$ is within distance $o(1)$ of either $(\sqrt{2} / 4,1 / 2, \sqrt{2} / 4,0, \sqrt{3} / 6)$ or $(\sqrt{2} / 4,-1 / 2, \sqrt{2} / 4,0, \sqrt{3} / 6)$. Hence $\operatorname{Dist}\left(S, S^{\prime}\right)=\sqrt{7 / 12}+o(1)$. Then $R\left(S \cup S^{\prime}\right)=$ $R(S) * R\left(S^{\prime}\right)$. By the Künneth Formula,

$$
\tilde{\beta}_{p}(R(S)) \geq\left(\left\lfloor\sqrt{\frac{m}{3}}\right\rfloor^{3}-m\right)\left(\left\lfloor\frac{m}{p-2}\right\rfloor-1\right)^{p / 2-1}=\Omega\left(n^{p / 2+1 / 2}\right) .
$$

\section{Quasi-Vietoris-Rips Complexes}

Quasi-Vietoris-Rips complexes, discussed by Chambers et al. [6], are relaxations of Vietoris-Rips complexes. Given a finite set $S \subset \mathbb{R}^{d}$ and a fixed $0<\alpha<1$, a quasiVietoris-Rips complex with parameter $\alpha$ on $S$ is a flag complex with vertex set $S$, an edge $u v$ whenever $\operatorname{dist}(u, v) \leq \alpha$, and no edge $u v$ when $\operatorname{dist}(u, v)>1$. If $\alpha<$ $\operatorname{dist}(u, v) \leq 1$, then $u v$ may be included or excluded arbitrarily. All Vietoris-Rips complexes are quasi-Vietoris-Rips complexes.

There is much greater freedom in the kinds of graphs that arise as the graphs of quasi-Vietoris-Rips complexes. Despite this freedom, the Betti numbers of quasiVietoris-Rips complexes obey nontrivial upper bounds. Let $M_{p, d}^{\alpha}(n)$ be the maximum value of $\tilde{\beta}_{p}(\Gamma)$, where $\Gamma$ is a Quasi-Vietoris-Rips complex with parameter $\alpha$ on a set of $n$ points in $\mathbb{R}^{d}$. In this section, we prove bounds on $M_{1, d}^{\alpha}(n), M_{2, d}^{\alpha}(n)$, and $M_{p, d}^{\alpha}(n)$. These bounds are analogous to the results of the previous sections.

Theorem 6.1 For all $d, n$, and $\alpha$, we have that

$$
M_{1, d}^{\alpha}(n) \leq\left(\left\lceil\frac{2 d^{1 / 2}}{\alpha}\right]^{d}-1\right) n=O(n) .
$$

Proof Consider $S \subset \mathbb{R}^{d}$ and $v \in S$. All vertices of $\operatorname{lk}(v)$ are contained in a ball $B$ of radius 1 , and there exists a set of $\left\lceil 2 d^{1 / 2} \alpha^{-1}\right\rceil^{d}$ cubes of side length $\alpha d^{-1 / 2}$ that covers $B$. Two vertices in the same cube have distance at most $\alpha$ and are joined by an edge. Hence $\operatorname{lk}(v)$ has at most $\left\lceil 2 d^{1 / 2} \alpha^{-1}\right\rceil^{d}$ connected components, and $\tilde{\beta}_{0}(\operatorname{lk}(v)) \leq$ $\left\lceil 2 d^{1 / 2} \alpha^{-1}\right\rceil^{d}-1$. The theorem follows by induction on $n$ and Lemma 2.1 .

Theorem 6.2 Fix $d \geq 2$ and $0<\alpha<1$. Then $M_{2, d}^{\alpha}(n)=\Theta(I(n))$.

Proof The proof of the upper bound is very similar to that of Theorem 4.7. The only change necessary is to use $\epsilon=\alpha d^{-1 / 2}$ instead of $\epsilon=d^{-1 / 2}$.

We prove the lower bound by construction. Assume that $n$ is a multiple of 3 , and consider a bipartite graph $G$ with vertex set $U \sqcup V$ with $|U|=|V|=n$ and a set $\mathcal{M}$ of $n$ disjoint, induced matchings on $G$ with $\sum_{M \in \mathcal{M}}|M|=I(n)$. Choose $\mathcal{M}^{\prime} \subset \mathcal{M}, U^{\prime} \subset U$, and $V^{\prime} \subset V$ uniformly at random among subsets of size $n / 3$, and restrict each element of $\mathcal{M}^{\prime}$ to edges with vertices in $U^{\prime}$ and $V^{\prime}$. Since each edge of $\bigcup_{M \in \mathcal{M}} M$ has probability $1 / 27$ of being in $\bigcup_{M \in \mathcal{M}^{\prime}} M$, the expected value of 
$\sum_{M \in \mathcal{M}^{\prime}}|M|$ is $\frac{1}{27} I(n)$, and so we assume that $\mathcal{M}^{\prime}, U^{\prime}$, and $V^{\prime}$ are chosen so that $\sum_{M \in \mathcal{M}^{\prime}}|M| \geq \frac{1}{27} I(n)$.

Let $G^{\prime}$ be the graph with vertex set identified with $U^{\prime} \sqcup V^{\prime} \sqcup \mathcal{M}^{\prime}$ and with edges exactly as follows. Let $G^{\prime}\left[U^{\prime}\right], G^{\prime}\left[V^{\prime}\right]$, and $G^{\prime}\left[\mathcal{M}^{\prime}\right]$ be cliques. For $u \in U^{\prime}$ and $v \in V^{\prime}$, say that $u v$ is an edge in $G^{\prime}$ if it is an edge in $G$. Also, say that $M u$ and $M v$ are edges in $G^{\prime}$ if $u v$ is an edge in the matching $M$. If $\Gamma$ is the unique flag complex with underlying graph $G^{\prime}$, then by Lemma 4.9 ,

$$
\tilde{\beta}_{2}(\Gamma) \geq \sum_{M \in \mathcal{M}^{\prime}}|M|-\left|U^{\prime}\right|-2\left|\mathcal{M}^{\prime}\right| \geq \frac{1}{27} I(n)-n .
$$

Finally, we show that $\Gamma$ can be realized as a quasi-Vietoris-Rips complex with parameter $\alpha$ in $\mathbb{R}^{2}$. Place all points of $U^{\prime}, V^{\prime}$, and $\mathcal{M}^{\prime}$ within distance $\alpha / 2$ of $(0,0)$, $(0,1)$, and $(\sqrt{3} / 2,1 / 2)$ respectively, with all points of $U^{\prime} \sqcup V^{\prime} \sqcup \mathcal{M}^{\prime}$ inside the triangle with vertices $(0,0),(0,1)$, and $(\sqrt{3} / 2,1 / 2)$.

Following the argument of Theorem 4.7, for $\epsilon=\alpha d^{-1 / 2}, r=3 / 2$, and large $n$, we have that

$$
M_{2, d}^{\alpha}(n) \leq 2\left(\begin{array}{c}
\kappa_{d, r, \epsilon} \\
2
\end{array}\right) I(n)+\left(C_{d, r, \epsilon}+\left(\begin{array}{c}
\kappa_{d, r, \epsilon} \\
2
\end{array}\right)\right) n
$$

Theorem 6.3 Fix $d \geq 2, p>2$, and $0<\alpha<1$. Then $M_{p, d}^{\alpha}(n)=O\left(n^{p-2} I(n)\right)$.

Proof The claim that $M_{p, d}^{\alpha}(n) \leq n M_{p-1, d}^{\alpha}(n)$ follows by Lemma 2.1, induction on $n$, and the fact that $M_{p, d}^{\alpha}(n)$ is non-decreasing in $n$, similarly to the claim in the proof of Theorem 5.1. The theorem then follows by Theorem 6.2 and induction on $p$.

\section{Concluding Remarks}

In general, while the upper and lower bounds on $M_{p, d}(n)$ presented in this paper are nontrivial, there are considerable gaps between the two. The author makes no conjectures beyond that which has already been proven. The value of $M_{p, 2}(n)$ has been determined to within a multiplicative constant for $p \leq 3\left(\Theta(n), \Theta(n)\right.$, and $\Theta\left(n^{2}\right)$ for $p=1,2$, and 3 respectively). Can the methods of Theorem 4.1 be adapted to estimate $M_{4,2}(n)$ ? It would also be interesting to know whether $M_{2,3}(n)$ and $M_{2,4}(n)$ grow linearly in $n$, as does $M_{2,2}(n)$.

Do the concepts of $\epsilon$-simple cycles and a pseudobasis have useful analogues in higher dimensions? The natural candidate for a two-dimensional version of an $\epsilon$-simple cycle, the octahedron, does not seem to work; the construction of Theorem 4.8 , while having large $\tilde{\beta}_{2}$ and points contained in a ball of radius slightly over $1 / 2$, does not have an induced octahedron. However, perhaps some slightly more complicated cycle would work. Also, we have expressed the value of $M_{p, d}^{\alpha}(n)$ to within a multiplicative constant of another natural combinatorial function, $I(n)$, when $p=2$. Can something similar be done for larger values of $p$ ? 
Vietoris-Rips complexes can be defined in general metric spaces. One possibility is to compare $M_{p, d}(n)$ to the bound that would result by considering Vietoris-Rips complexes on a given $d$-dimensional Riemannian manifold.

One may also consider extremal Betti numbers of C̆ech complexes, another tool for analyzing the topology of a point cloud. Given $S \subset \mathbb{R}^{d}$, the $\check{C}$ ech complex $C^{\delta}(S)$ has vertex set $S$ and a face $F$ whenever $F$ is contained in a ball of radius $\delta / 2$. By the Nerve Lemma [19], $\tilde{\beta}_{p}\left(C^{\delta}(S)\right)=0$ for $p \geq d$, in contrast to the situation for Vietoris-Rips complexes. A challenge of extending our results to Čech complexes is that if $\Gamma$ is a Čech complex and $v \in \mathcal{V}(\Gamma)$, then $1 \mathrm{k}(v)$ might not be a C̆ech complex.

Acknowledgements The author wishes to thank Matt Kahle for suggesting the problem and providing many helpful conversations. This research was partially supported by Grant DMS-0801152.

\section{References}

1. Björner, A., Kalai, G.: On $f$-vectors and homology. In: Combinatorial Mathematics: Proceedings of the Third International Conference, New York, 1985. Ann. New York Acad. Sci., vol. 555, pp. 63-80. New York Acad. Sci., New York (1989)

2. Carlsson, G., De Silva, V.: Topological estimation using witness complexes. In: SPBG Symposium on Point-Based Graphics, pp. 157-166 (2004)

3. Carlsson, G., Collins, A., Guibas, L., Zomorodian, A.: Persistence barcodes for shapes. Int. J. Shape Model. 11, 149-187 (2005)

4. Carlsson, E., Carlsson, G., De Silva, V.: An algebraic topological method for feature identification. Int. J. Comput. Geom. Appl. 16, 291-314 (2006)

5. Carlsson, G., De Silva, V., Ishkhanov, T., Zomorodian, A.: On the local behavior of spaces of natural images. Int. J. Comput. Vis. 71, 1-12 (2008)

6. Chambers, E., De Silva, V., Erickson, J., Ghrist, R.: Rips complexes of planar point sets. Discrete Comput. Geom. 44(1), 75-90 (2010)

7. Chazal, F., Oudot, S.: Towards persistence-based reconstruction in Euclidean spaces. In: Proc. 2008 ACM Symposium of Computational Geometry

8. De Silva, V., Ghrist, R.: Coordinate-free coverage in sensor networks with controlled boundaries via homology. Int. J. Robot. Res. 25, 1205-1222 (2006)

9. De Silva, V., Ghrist, R.: Coverage in sensor networks via persistent homology. Algebraic Geom. Topol. 7, 339-358 (2007)

10. Elkin, M.: An improved construction of progression-free sets. arXiv:0801.4310v1 [math.NT]

11. Gromov, M.: Hyperbolic groups. In: Essays in Group Theory. MSRI Publ., vol. 8. Springer, Berlin (1987)

12. Hatcher, A.: Algebraic Topology. Cambridge University Press, Cambridge (2002)

13. Hausmann, J.: On the Vietoris-Rips complexes and a cohomology theory for metric spaces. In: Prospects in Topology: Proceedings of a Conference in Honour of William Browder. Ann. of Math. Stud., vol. 138, pp. 175-188. Princeton Univ. Press, Princeton (1995)

14. Jadbabaie, A., Muhammad, A.: Dynamic coverage verification in mobile sensor networks via switched higher order Laplacians. Robot. Sci. Syst. (2007)

15. Kahle, M.: Random geometric complexes. arXiv:0910.1649v1 [math.PR]

16. Kahle, M.: Topology of random clique complexes. Discrete Math. 309(6), 1658-1671 (2009)

17. Kövári, T., Sós, V., Turán, P.: On a problem of K. Zarankiewicz. Colloq. Math. 3, 50-57 (1954)

18. Latschev, J.: Vietoris-Rips complexes of metric spaces near a closed Riemannian manifold. Arch. Math. 77(6), 522-528 (2001)

19. Leray, J.: Sur la forme des espaces topologiques et sur les points fixes des représentations. J. Math. 24, 95-167 (1945) 
20. Pfender, F., Ziegler, G.: Kissing numbers, sphere packings, and some unexpected proofs. Not. Am. Math. Soc. 51, 873-883 (2004)

21. Ruzsa, I., Szemerédi, E.: Triple systems with no six points carrying three triangles. In: Combinatorics, vol. II. Colloq. Math. Soc. J. Bolyai, vol. 18, pp. 939-994. North-Holland, Amsterdam (1978)

22. Tao, T., Vu, V.: Additive Combinatorics. Cambridge Univ. Press, Cambridge (2006)

23. Vietoris, L.: Über den höheren Zusammenhang kompaktor Räume und eine Klasse von zusammenhangstreuen Abbildungen. Math. Ann. 97, 454-472 (1927) 